\title{
Adopting Scenario Based Learning in Critical Care Nursing Education: Students’ Achievement and Feedback
}

\author{
Hanaa Hussein Ahmed* \\ Critical Care and Emergency Nursing Department, Mansoura University, Egypt \\ *Corresponding author: hanatop.99@gmail.com
}

Received April 14, 2019; Revised May 20, 2019; Accepted June 04, 2019

\begin{abstract}
Scenario based learning (SBL) is up-to-date inventive teaching method that providing directive discussion for nursing students. Effective designed scenarios are considered the corner stone for teachers to integrate learning objectives into nursing students' learning experience. Many studies reported that SBL is a broad dynamic interactive teaching and learning strategy. It enhances students' decision making skills and self-learning abilities. Aim: the current study aimed to explore fourth level nursing students' achievement and feedback about scenario based learning at Faculty of Nursing, Mansoura University. Method: a descriptive research design was used. A total of 251 fourth level nursing students registered in critical care nursing course in the second semester of the academic year 2016-2017 were involved in the study. Two tools were used to collect data for the present study which was developed by the researcher. Tool one "Students' Achievement Sheet" that involved eight main domains. Tool two is "Students' Feedback about SBL" which folded 18 statements. Results: The majority of the students analyzed scenarios clearly by discovering the cues and inferences related to the scenario problem with high score ranged from good to excellent (61.25-96.25). Despite around 70\% of the students' perceived SBL as a very stressful learning method, it was exciting and enjoyable (62.7\%) for them. Also, most of them (75\%) agreed that the teachers were a good guide and facilitator for them. Conclusion: The findings of the current study support the argument that SBL is an effective and dynamic learning method and easy to be conducted. It enhances the nursing students' skills and learning abilities to bridge the theory-practice gap.
\end{abstract}

Keywords: scenario based learning, critical care nursing education, students' achievement, students' feedback

Cite This Article: Hanaa Hussein Ahmed, "Adopting Scenario Based Learning in Critical Care Nursing Education: Students’ Achievement and Feedback.” American Journal of Nursing Research, vol. 7, no. 4 (2019): 581-588. doi: 10.12691/ajnr-7-4-20.

\section{Introduction}

The vast development in information technologies and communication means has a great influence on education process and teaching strategies [1,2]. In the last decades, a lot of recent teaching strategies are produced to face the quick technological development $[2,3,4]$. One of these strategies is SBL which takes a significant attraction recently from both teachers and learners [2,5]. The area of SBL is a wide-ranging especially in nursing field [6]. SBL is a modern creative teaching fashion that improves nursing students' learning experiences [7]. The traditional teaching strategies enroll the curriculum or teacher center approach while, SBL adopts student centered approach $[8,9]$.

According to Akins \& Crichton [10], SBL uniforms problems that may have numerous explanations. Pervious and recent studies pointed that SBL can be folded main four types of scenarios such as professional debate scenarios, professional hypothetical scenarios, scenarios conveying skills and knowledge, and scenarios to inquiry problems [11-14]. Impactful designed scenarios afford the core for teachers to confront learning objectives and deliver a significant learning experience for nursing students $[6,15]$.

Many researchers stated that SBL is a vastly dynamic interactive teaching and learning strategy $[14,16,17]$. This strategy enriches nursing students with various learning objectives to help them bridging the theory-practical gab $[18,19,20]$. Additionally, many authors emphasized that SBL focuses mainly on students' abilities and skills' enhancement including analytic, intellectual, problem-solving, communication, and teamwork skills which is the $21^{\text {st }}$ Century skills [21,22,23]. Moreover, it improves students' empowerment and self-confidence [13,24,25]. It has reported that SBL can help students to think critically and enhance their decision making skills and self-learning abilities [11,26,27].

Nursing education stakeholders are facing huge challenges to introduce high quality nursing programs that attributing graduate nurses who are able to competing in the job marketing $[15,27,28]$. Nursing education in Egypt 
has various programs [29]. One of them is nursing Bachelor degree that introduces many courses to equip nursing students with required knowledge and skills for graduation [30]. Critical care nursing course is one of these courses at Faculty of Nursing, Mansoura University that is studied through level four. The duration of the theoretical bulk encompassed fifteen weeks.

\subsection{Significance of the Study}

Nursing teachers have great challenges to develop students thinking abilities, and to transform their knowledge into nursing interventions [31]. Lacking of learning chances among critical care nursing students in both lectures and clinical training is the biggest issue that needs unusual approaches or strategies [13]. SBL in critical care nursing education delivers an alternative approach to the traditional style that focuses on students' learning needs and favorites [2,16,27]. This strategy can confer students to disclose their learning experiences [32,33,34]. There are many studies that examined other learning and teaching strategies such as problem based learning in many other nursing courses. On the contrary, SBL research studies in critical care nursing are scarce especially in Egypt. Thus, this study was conducted to explore the effectiveness of this strategy for nursing students who study critical care nursing courses.

\subsection{Aim of the Study}

The current study aimed to explore fourth level nursing students' achievement and feedback about SBL at Faculty of Nursing, Mansoura University.

\subsection{Research Questions}

1. What is the fourth level nursing students' achievement when adopting SBL in critical care nursing education?

2. What is the fourth level nursing students' feedback about SBL as a learning strategy?

\section{Subjects and Methods}

\subsection{Research Design}

A descriptive research design was used to collect data regarding fourth level nursing students' achievement and feedback about SBL used in critical care nursing course.

\subsection{Participants}

All fourth level nursing students who were registered in critical care nursing course $(n=251)$ in the second semester of the academic year 2016-2017 were involved in the study.

\subsection{Setting}

The study was taken place in critical care and emergency nursing department, Faculty of Nursing, Mansoura University, Egypt.

\subsection{Ethical Considerations}

Ethical approval was taken from the Research Ethics Committee of Faculty of Nursing, Mansoura University. Further approval was gained from the Dean of the Faculty. An informed consent element was involved within the questionnaire. The participants were assured that their participation in the study was voluntary. Questionnaire completion and submission reflects students' agreement to participate in the study. They were assured that their personal information would be coded and kept confidential.

\subsection{Instrument}

Two tools were used to collect data for the present study which was developed by the researcher based up on relevant literature. Tool one "Students' Achievement Sheet” [13,18,24-29,35] that involved eight main domains. The first domain comprised 4 statements about analyzing the scenario. The second domain covered 4 statements about students' intellectual skills. The third one involved 3 statements about students' critical appraisal. Using learning resources and presentation skills domains have 4 statements for each. Additionally, writing criteria encompassed 5 statements. Students' communication skills and language domain included 5 statements. Group work is the last domain that covered 4 statements. Each item of this tool was checked on a five-point Likert scale (1 = "unachieved", 2 = "fair", 3 = "good", 4 = "very good" and 5 = "excellent”). Tool two "Students' Feedback about Scenario Based Learning" which folded 18 statements $[2,6,13,24,27,29]$. It is focused on the students' evaluation for using SBL as teaching strategy, teacher guidance and their overall satisfaction. Students responded to each items by using a five-point Likert scale ( 1 = "strongly disagree", 2 = "disagree", 3 = "confused", 4 = "agree" and 5 = "strongly agree”). Content validity of the tools was tested by 5 experts from critical care and emergency nursing department, Faculty of Nursing, Mansoura University. The tools have been revealed to be highly reliable with a Cronbach's alpha of 0.930 . A pilot study was conducted on 23 students who were excluded from the study sample. It was applied to evaluate the tools clarity and applicability and modifications were done accordingly.

\subsection{Data Collection}

Students were registered in critical care nursing course through the first week of the semester. Topics for scenarios were selected (acute lung failure and mechanical ventilation). Scenarios were prepared by the researcher within two weeks. Students were informed about the nature of the study. Then, they were assigned into small groups (19) about 12 students in each. Each group was guided by one teacher. All groups were informed about the required references from faculty library and database. Prepared Scenarios were presented to all groups within four sessions. Each session was taken about 15 to 20 minutes. Each group was instructed to search and prepare the required knowledge according to the cusses and inferences of scenarios in a time plan of 7 weeks 
(Figure 1). At the end, each group presented the data within 5 to 10 minutes. Students' achievement in each group was evaluated by the teacher during and after each session using tool one. Lastly, students evaluated the SBL strategy and their teachers' role at the last week using tool two.

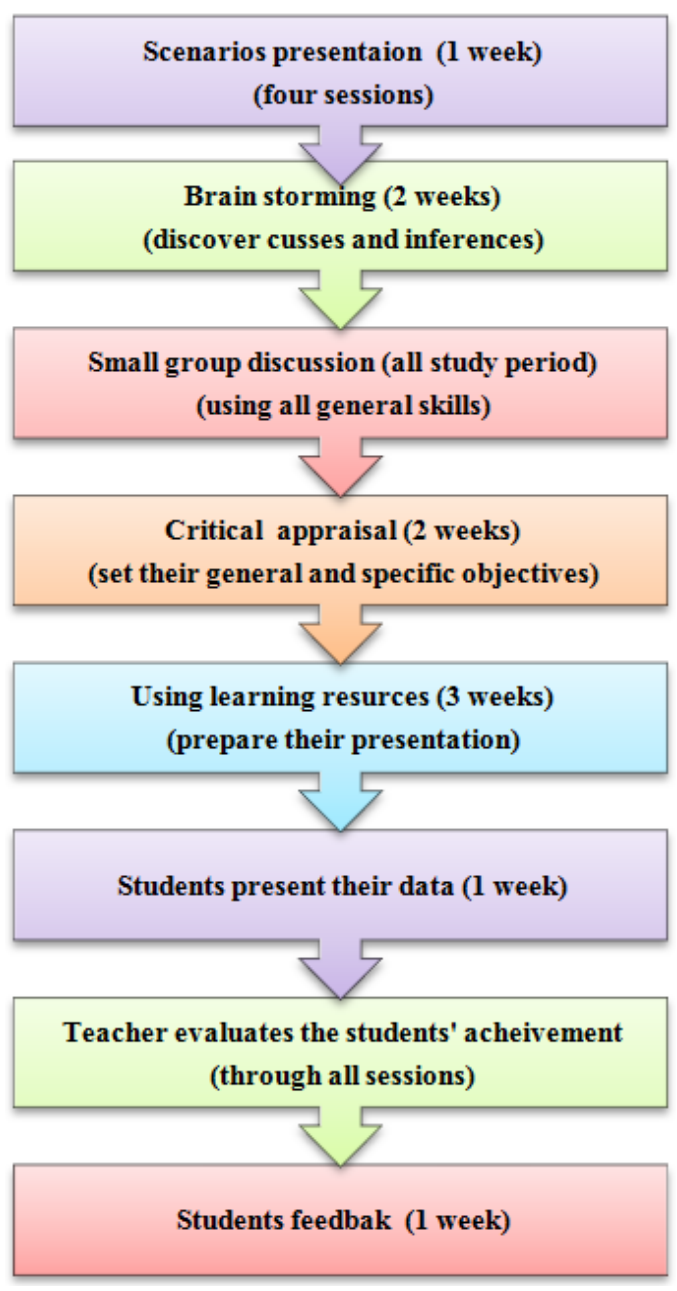

Figure 1. Application of Scenario Based Learning

\subsection{Data Analysis}

The Statistical Package for Social Science version 25.0 (SPSS, Chicago, IL, USA) was used to analyze the collected data. Quantitative data were presented as median and interquartile range (IQR). Qualitative data were presented as count and percentage and compared by one sample Chi- Square test. Results were considered as statistically significant when $\mathrm{p}$ value $\leq 0.050$. Figures or charts were used to describe the data whenever required.

\section{Results}

This study involved 228 students in the second semester of 2016/2017. Ten students (8.8\%) didn't declare their gender. The majority of the participants were females $75.9 \%$ while, males represents $24.1 \%$ with no statistically significant difference in gender distribution.

Table 1: summarizes nursing students' achievement when adopting SBL in critical care nursing course. The majority of the students analyzed scenarios clearly by discovering the cusses and inferences related to the scenario problem with high score ranged from very good to excellent (61.25-96.25). Additionally, most of the students delivered their presentation skillfully (75-90) with effective group communication (80-90) and team work to achieve their duties (80-90). However, most of them gave a good achievement regarding intellectual skills (70-80) and using the available learning resources effectively (72-88). Conversely, the lowest students' achievement was seen in their critical appraisal of obtained information (60-73.3) and their writing criteria (52-80). In general, these results answered the first research question that SBL has a positive effect on the students' achievement in critical care nursing education.

Students' feedback about the application of SBL is presented in Table 2. The vast majority of them agreed that SBL was effective to achieve their learning objectives (77.6\%), gave them a realistic knowledge in critical care nursing field and encouraged them to participate in the learning process (75.4\% for both). Despite around 70\% of the students' perceived SBL as a very stressful learning method, but it was exciting and enjoyable (62.7\%) for them. This is because it promoted their abilities to work a team (60.5\%), motivated them to do the best (61.4\%), and improved their analytic (68\%) and searching (65.8\%) skills for various information resources. Overall, more than $60 \%$ of the students were satisfied with the SBL method in critical care education. However, a high statistical significant difference was recorded in all items of students' feedback about SBL strategy.

Table 3: depicts students' feedback about teachers' role and guidance during the application of SBL. Most of students agreed that teachers stimulated them to analyze scenario scientifically (75.9), link scenario data with the discussed issue (73.7), integrate knowledge with the patient care (74.1) and discover cusses and inferences from the scenario (75.9). However, slightly more than $60 \%$ of them reported that teachers motivated them to summarize what they had learnt. Highly statistical significance difference was noted regarding students' feedback about teachers' role and guidance.

Table 1. Students' achievement when adopting SBL in critical care nursing course

\begin{tabular}{|c|c|c|c|c|c|c|}
\hline \multirow{2}{*}{ Domain } & \multicolumn{5}{|c|}{ Students' achievement score $(n=228)$} & \multirow{2}{*}{ Total Median (IQR) } \\
\hline & $\mathbf{U}$ & $\mathbf{F}$ & G & VG & $\mathbf{E}$ & \\
\hline \multicolumn{6}{|l|}{ Analyze scenario } & \multirow{5}{*}{$\begin{array}{c}80 \\
(61.25-96.25)\end{array}$} \\
\hline 1. Analyze the scenario problem clearly & $\begin{array}{c}3 \\
(1.3)\end{array}$ & $\begin{array}{c}25 \\
(11.0)\end{array}$ & $\begin{array}{c}38 \\
(16.7)\end{array}$ & $\begin{array}{c}104 \\
(45.6)\end{array}$ & $\begin{array}{c}58 \\
(25.4)\end{array}$ & \\
\hline $\begin{array}{l}\text { 2. Discover cusses and inferences from the } \\
\text { scenario }\end{array}$ & $\begin{array}{c}2 \\
(0.9) \\
\end{array}$ & $\begin{array}{c}34 \\
(14.9) \\
\end{array}$ & $\begin{array}{c}43 \\
(18.9) \\
\end{array}$ & $\begin{array}{c}91 \\
(39.9) \\
\end{array}$ & $\begin{array}{c}58 \\
(25.4) \\
\end{array}$ & \\
\hline 3. Determine the main causes of the problem & $\begin{array}{c}7 \\
(3.1)\end{array}$ & $\begin{array}{c}13 \\
(5.7)\end{array}$ & $\begin{array}{c}56 \\
(24.6)\end{array}$ & $\begin{array}{c}94 \\
(41.2)\end{array}$ & $\begin{array}{c}58 \\
(25.4)\end{array}$ & \\
\hline $\begin{array}{l}\text { 4. Mention the differential diagnosis related to } \\
\text { the scenario }\end{array}$ & $\begin{array}{c}9 \\
(3.9)\end{array}$ & $\begin{array}{c}11 \\
(4.8)\end{array}$ & $\begin{array}{c}64 \\
(28.1)\end{array}$ & $\begin{array}{c}86 \\
(37.7)\end{array}$ & $\begin{array}{c}58 \\
(25.4)\end{array}$ & \\
\hline
\end{tabular}




\begin{tabular}{|c|c|c|c|c|c|c|}
\hline \multirow{2}{*}{ Domain } & \multicolumn{5}{|c|}{ Students' achievement score $(n=228)$} & \multirow{2}{*}{ Total Median (IQR) } \\
\hline & $\mathbf{U}$ & $\mathbf{F}$ & G & VG & $\mathbf{E}$ & \\
\hline \multicolumn{6}{|l|}{ Intellectual skills } & \multirow{5}{*}{$75(70-80)$} \\
\hline 1. Brain storming process & $\begin{array}{c}8 \\
(3.5)\end{array}$ & $\begin{array}{c}13 \\
(5.7)\end{array}$ & $\begin{array}{c}91 \\
(39.9)\end{array}$ & $\begin{array}{c}94 \\
(41.2)\end{array}$ & $\begin{array}{c}22 \\
(9.6)\end{array}$ & \\
\hline 2. Organize learning objectives & $\begin{array}{c}8 \\
(3.5)\end{array}$ & $\begin{array}{c}9 \\
(3.9)\end{array}$ & $\begin{array}{c}41 \\
(18)\end{array}$ & $\begin{array}{c}136 \\
(59.6)\end{array}$ & $\begin{array}{c}34 \\
(14.9)\end{array}$ & \\
\hline $\begin{array}{l}\text { 3. Priotorize main care related to the scenario } \\
\text { problem }\end{array}$ & $\begin{array}{l}0.0 \\
(0.0)\end{array}$ & $\begin{array}{c}18 \\
(7.9)\end{array}$ & $\begin{array}{c}19 \\
(8.3)\end{array}$ & $\begin{array}{c}162 \\
(71.1)\end{array}$ & $\begin{array}{c}29 \\
(12.7)\end{array}$ & \\
\hline Critical thinking process & $\begin{array}{c}3 \\
(1.3) \\
\end{array}$ & $\begin{array}{c}9 \\
(3.9)\end{array}$ & $\begin{array}{c}101 \\
(44.3)\end{array}$ & $\begin{array}{c}103 \\
(45.2)\end{array}$ & $\begin{array}{c}12 \\
(9.2) \\
\end{array}$ & \\
\hline \multicolumn{6}{|l|}{ Critical appraisal } & \multirow{4}{*}{$66.7(60-73.3)$} \\
\hline 1. Propose the issues outline & $\begin{array}{l}0.0 \\
(0.0)\end{array}$ & $\begin{array}{c}19 \\
(8.3)\end{array}$ & $\begin{array}{c}57 \\
(25.0)\end{array}$ & $\begin{array}{c}131 \\
(57.5)\end{array}$ & $\begin{array}{c}21 \\
(9.2)\end{array}$ & \\
\hline 2. Motivated, initiated, and have curiosity & $\begin{array}{c}9 \\
(3.9)\end{array}$ & $\begin{array}{c}9 \\
(3.9)\end{array}$ & $\begin{array}{c}74 \\
(32.5)\end{array}$ & $\begin{array}{c}127 \\
(55.7)\end{array}$ & $\begin{array}{c}9 \\
9 \\
(3.9)\end{array}$ & \\
\hline 3. Criticize the information constructively & $\begin{array}{c}16 \\
(7.0)\end{array}$ & $\begin{array}{c}37 \\
(16.2) \\
\end{array}$ & $\begin{array}{c}134 \\
(58.8)\end{array}$ & $\begin{array}{c}33 \\
(44.5) \\
\end{array}$ & $\begin{array}{c}8 \\
(3.5)\end{array}$ & \\
\hline \multicolumn{6}{|l|}{ Learning resources } & \multirow{6}{*}{$80(72-88)$} \\
\hline 1. Select the relevant resources & $\begin{array}{c}0.0 \\
(0.0)\end{array}$ & $\begin{array}{c}0.0 \\
(0.0)\end{array}$ & $\begin{array}{c}18 \\
(7.9)\end{array}$ & $\begin{array}{c}145 \\
(63.6)\end{array}$ & $\begin{array}{c}65 \\
(28.5) \\
\end{array}$ & \\
\hline 2. Utilize the resources effectively & $\begin{array}{c}0.0 \\
(0.0)\end{array}$ & $\begin{array}{c}0.0 \\
(0.0) \\
\end{array}$ & $\begin{array}{c}23 \\
(10.1) \\
\end{array}$ & $\begin{array}{c}130 \\
(57.0)\end{array}$ & $\begin{array}{c}75 \\
(32.9) \\
\end{array}$ & \\
\hline $\begin{array}{l}\text { 3. Use the appropriate knowledge to the } \\
\text { scenario }\end{array}$ & $\begin{array}{c}0.0 \\
(0.0) \\
\end{array}$ & $\begin{array}{c}7 \\
(3.1) \\
\end{array}$ & $\begin{array}{c}58 \\
(25.4) \\
\end{array}$ & $\begin{array}{c}88 \\
(38.6) \\
\end{array}$ & $\begin{array}{c}75 \\
(32.9) \\
\end{array}$ & \\
\hline $\begin{array}{l}\text { 4. Cover all inferences of the scenario with } \\
\text { complete and adherent knowledge }\end{array}$ & $\begin{array}{c}0.0 \\
(0.0)\end{array}$ & $\begin{array}{c}0.0 \\
(0.0)\end{array}$ & $\begin{array}{c}85 \\
(37.3)\end{array}$ & $\begin{array}{c}116 \\
(50.9)\end{array}$ & $\begin{array}{c}27 \\
(11.8)\end{array}$ & \\
\hline $\begin{array}{l}\text { 5. Use different resources as figures, } \\
\text { text...etc., }\end{array}$ & $\begin{array}{c}7 \\
(3.1) \\
\end{array}$ & $\begin{array}{c}0.0 \\
(0.0) \\
\end{array}$ & $\begin{array}{c}50 \\
(21.9) \\
\end{array}$ & $\begin{array}{c}148 \\
(64.9) \\
\end{array}$ & $\begin{array}{c}23 \\
(10.1) \\
\end{array}$ & \\
\hline \multicolumn{6}{|l|}{ Presentation skills } & \multirow{5}{*}{$85(75-90)$} \\
\hline 1. Use internet effectively & $\begin{array}{c}0.0 \\
(0.0)\end{array}$ & $\begin{array}{c}10 \\
(4.4)\end{array}$ & $\begin{array}{c}58 \\
(25.4) \\
\end{array}$ & $\begin{array}{c}106 \\
(46.5) \\
\end{array}$ & $\begin{array}{c}54 \\
(23.7) \\
\end{array}$ & \\
\hline 2. Use presentation tools effectively & $\begin{array}{c}18 \\
(7.9)\end{array}$ & $\begin{array}{c}0.0 \\
(0.0) \\
\end{array}$ & $\begin{array}{c}8 \\
(3.5)\end{array}$ & $\begin{array}{c}44 \\
(19.3) \\
\end{array}$ & $\begin{array}{c}158 \\
(69.3) \\
\end{array}$ & \\
\hline 3. Committed with presentation time & $\begin{array}{c}18 \\
(7.9) \\
\end{array}$ & $\begin{array}{c}0.0 \\
(0.0) \\
\end{array}$ & $\begin{array}{c}1 \\
(0.9) \\
\end{array}$ & $\begin{array}{c}118 \\
(51.8) \\
\end{array}$ & $\begin{array}{c}91 \\
(39.9) \\
\end{array}$ & \\
\hline 4. Committed with PowerPoint guidelines & $\begin{array}{c}35 \\
(15.4)\end{array}$ & $\begin{array}{c}0.0 \\
(0.0)\end{array}$ & $\begin{array}{c}64 \\
(20.2) \\
\end{array}$ & $\begin{array}{c}124 \\
(54.4)\end{array}$ & $\begin{array}{c}23 \\
(10.1)\end{array}$ & \\
\hline \multicolumn{6}{|l|}{ Writing criteria } & \multirow{6}{*}{$72(52-80)$} \\
\hline 1. Complete and organized outline & $\begin{array}{c}0.0 \\
(0.0)\end{array}$ & $\begin{array}{c}38 \\
(16.7) \\
\end{array}$ & $\begin{array}{c}26 \\
(11.4) \\
\end{array}$ & $\begin{array}{c}103 \\
(45.2) \\
\end{array}$ & $\begin{array}{c}61 \\
(26.8) \\
\end{array}$ & \\
\hline $\begin{array}{l}\text { 2. Organized, relevant and coherent data and } \\
\text { information }\end{array}$ & $\begin{array}{l}0.0 \\
(0.0)\end{array}$ & $\begin{array}{c}53 \\
(23.2)\end{array}$ & $\begin{array}{c}11 \\
(4.8)\end{array}$ & $\begin{array}{c}129 \\
(56.6)\end{array}$ & $\begin{array}{c}35 \\
(15.4)\end{array}$ & \\
\hline $\begin{array}{l}\text { 3. Committed with an academic writing } \\
\text { criteria }\end{array}$ & $\begin{array}{c}24 \\
(10.5) \\
\end{array}$ & $\begin{array}{c}29 \\
(12.7) \\
\end{array}$ & $\begin{array}{c}102 \\
(44.7) \\
\end{array}$ & $\begin{array}{c}70 \\
(30.7) \\
\end{array}$ & $\begin{array}{c}3 \\
(1.3) \\
\end{array}$ & \\
\hline 4. Complete and correct references written & $\begin{array}{c}28 \\
(12.3) \\
\end{array}$ & $\begin{array}{c}27 \\
(11.8) \\
\end{array}$ & $\begin{array}{c}128 \\
(56.1)\end{array}$ & $\begin{array}{c}45 \\
(19.7) \\
\end{array}$ & $\begin{array}{c}0.0 \\
(0.0) \\
\end{array}$ & \\
\hline $\begin{array}{l}\text { 5. Coherent between presentation and data } \\
\text { written }\end{array}$ & $\begin{array}{c}37 \\
(16.2) \\
\end{array}$ & $\begin{array}{c}17 \\
(7.5) \\
\end{array}$ & $\begin{array}{c}83 \\
(36.4) \\
\end{array}$ & $\begin{array}{c}87 \\
(38.2) \\
\end{array}$ & $\begin{array}{c}4 \\
(1.8) \\
\end{array}$ & \\
\hline \multicolumn{6}{|l|}{ Communication skills \& language } & \multirow{5}{*}{$85(80-90)$} \\
\hline 1. Oral expression is correct and clear & $\begin{array}{c}0.0 \\
(0.0) \\
\end{array}$ & $\begin{array}{c}0.0 \\
(0.0) \\
\end{array}$ & $\begin{array}{c}18 \\
(7.9)\end{array}$ & $\begin{array}{c}75 \\
(32.9) \\
\end{array}$ & $\begin{array}{c}135 \\
(59.2) \\
\end{array}$ & \\
\hline 2. Provide and accept feedback & $\begin{array}{c}0.0 \\
(0.0)\end{array}$ & $\begin{array}{c}8 \\
(3.5) \\
\end{array}$ & $\begin{array}{c}38 \\
(16.7) \\
\end{array}$ & $\begin{array}{c}102 \\
(44.7) \\
\end{array}$ & $\begin{array}{c}80 \\
(35.1) \\
\end{array}$ & \\
\hline 3. Use communication resources effectively & $\begin{array}{l}0.0 \\
(0.0) \\
\end{array}$ & $\begin{array}{c}0.0 \\
(0.0)\end{array}$ & $\begin{array}{c}18 \\
(7.9)\end{array}$ & $\begin{array}{c}118 \\
(51.8) \\
\end{array}$ & $\begin{array}{c}92 \\
(40.4) \\
\end{array}$ & \\
\hline 4. Be confident enough during presentation & $\begin{array}{c}0.0 \\
(0.0)\end{array}$ & $\begin{array}{c}1 \\
(0.4)\end{array}$ & $\begin{array}{c}53 \\
(23.2) \\
\end{array}$ & $\begin{array}{c}160 \\
(70.2)\end{array}$ & $\begin{array}{c}14 \\
(6.1)\end{array}$ & \\
\hline \multicolumn{6}{|l|}{ Group work } & \multirow{5}{*}{$85(80-90)$} \\
\hline 1. Contribute to group harmony & $\begin{array}{c}20 \\
(8.8)\end{array}$ & $\begin{array}{c}10 \\
(4.4)\end{array}$ & $\begin{array}{c}19 \\
(8.3)\end{array}$ & $\begin{array}{c}88 \\
(38.6)\end{array}$ & $\begin{array}{c}91 \\
(39.9)\end{array}$ & \\
\hline 2. Organize and prepare for group discussion & $\begin{array}{c}0.0 \\
(0.0)\end{array}$ & $\begin{array}{c}19 \\
(8.3)\end{array}$ & $\begin{array}{c}36 \\
(15.8) \\
\end{array}$ & $\begin{array}{c}107 \\
(46.9)\end{array}$ & $\begin{array}{c}66 \\
(28.9) \\
\end{array}$ & \\
\hline $\begin{array}{l}\text { 3. Share opinions and thoughts with peers } \\
\text { actively }\end{array}$ & $\begin{array}{c}11 \\
(4.8)\end{array}$ & $\begin{array}{c}1 \\
(0.4)\end{array}$ & $\begin{array}{c}36 \\
(15.8) \\
\end{array}$ & $\begin{array}{c}111 \\
(48.7)\end{array}$ & $\begin{array}{c}69 \\
(30.3) \\
\end{array}$ & \\
\hline $\begin{array}{l}\text { 4. Share and discuss all information sources } \\
\text { with peers }\end{array}$ & $\begin{array}{c}9 \\
(3.9)\end{array}$ & $\begin{array}{c}9 \\
(3.9)\end{array}$ & $\begin{array}{c}8 \\
(3.5)\end{array}$ & $\begin{array}{c}21 \\
(9.2)\end{array}$ & $\begin{array}{c}181 \\
(79.4)\end{array}$ & \\
\hline
\end{tabular}

U: Unachieved F: Fair G: Good VG: Very good E: Excellent Data are presented as count and percentage IQR: Interquartile Range. 
Table 2. Students' feedback about the application of SBL in critical care nursing course

\begin{tabular}{|c|c|c|c|c|c|c|c|c|c|}
\hline \multirow{2}{*}{ Domain } & \multicolumn{5}{|c|}{ Students' feedback $(n=228)$} & \multicolumn{2}{|c|}{ Total } & \multicolumn{2}{|c|}{ Significance } \\
\hline & SD & D & C & A & SA & D & $\mathbf{A}$ & $\chi^{2}$ & $p$ \\
\hline $\begin{array}{l}\text { 1. SBL is more effective in achieving } \\
\text { learning objectives. }\end{array}$ & $\begin{array}{c}10 \\
(4.4)\end{array}$ & $\begin{array}{c}23 \\
(10.1)\end{array}$ & $\begin{array}{c}18 \\
(7.9)\end{array}$ & $\begin{array}{c}91 \\
(39.9)\end{array}$ & $\begin{array}{c}86 \\
(37.7)\end{array}$ & $\begin{array}{c}51 \\
(22.4)\end{array}$ & $\begin{array}{c}177 \\
(77.6)\end{array}$ & 96.632 & $<\mathbf{0 . 0 0 0 5}$ \\
\hline $\begin{array}{l}\text { 2. SBL gives best realistic knowledge } \\
\text { of critical care nursing. }\end{array}$ & $\begin{array}{c}8 \\
(3.5)\end{array}$ & $\begin{array}{c}18 \\
(7.9)\end{array}$ & $\begin{array}{c}30 \\
(13.2)\end{array}$ & $\begin{array}{l}104 . \\
(45.6)\end{array}$ & $\begin{array}{c}68 \\
(29.8)\end{array}$ & $\begin{array}{c}56 \\
(24.6)\end{array}$ & $\begin{array}{c}172 \\
(75.4)\end{array}$ & 59.018 & $<\mathbf{0 . 0 0 0 5}$ \\
\hline $\begin{array}{l}\text { 3. SBL encourages students to } \\
\text { participate in the learning process }\end{array}$ & $\begin{array}{c}6 \\
(2.6) \\
\end{array}$ & $\begin{array}{c}24 \\
(10.5)\end{array}$ & $\begin{array}{c}26 \\
(11.4)\end{array}$ & $\begin{array}{c}112 \\
(49.1) \\
\end{array}$ & $\begin{array}{c}60 \\
(26.3)\end{array}$ & $\begin{array}{c}56 \\
(24.6)\end{array}$ & $\begin{array}{c}172 \\
(75.4)\end{array}$ & 59.018 & $<\mathbf{0 . 0 0 0 5}$ \\
\hline 4. SBL is more exciting and enjoyable & $\begin{array}{c}9 \\
(3.9) \\
\end{array}$ & $\begin{array}{c}36 \\
(15.8) \\
\end{array}$ & $\begin{array}{c}40 \\
(17.5) \\
\end{array}$ & $\begin{array}{c}80 \\
(35.1) \\
\end{array}$ & $\begin{array}{c}63 \\
(27.6) \\
\end{array}$ & $\begin{array}{c}85 \\
(37.3) \\
\end{array}$ & $\begin{array}{c}143 \\
(62.7) \\
\end{array}$ & 14.754 & $<\mathbf{0 . 0 0 0 5}$ \\
\hline $\begin{array}{l}\text { 5. SBL encourages student's team } \\
\text { work and acquires interpersonal skills. }\end{array}$ & $\begin{array}{c}11 \\
(4.8)\end{array}$ & $\begin{array}{c}24 \\
(10.5)\end{array}$ & $\begin{array}{c}38 \\
(16.7) \\
\end{array}$ & $\begin{array}{c}93 \\
(40.8) \\
\end{array}$ & $\begin{array}{c}62 \\
(27.2)\end{array}$ & $\begin{array}{c}73 \\
(32.0)\end{array}$ & $\begin{array}{c}155 \\
(68.0) \\
\end{array}$ & 29.491 & $<\mathbf{0 . 0 0 0 5}$ \\
\hline $\begin{array}{l}\text { 6. SBL has helped me to develop } \\
\text { problem-solving skills related to } \\
\text { critical care nursing. }\end{array}$ & $\begin{array}{c}7 \\
(3.1)\end{array}$ & $\begin{array}{c}33 \\
(14.5)\end{array}$ & $\begin{array}{c}53 \\
(23.2)\end{array}$ & $\begin{array}{c}86 \\
(37.7)\end{array}$ & $\begin{array}{c}49 \\
(21.5)\end{array}$ & $\begin{array}{c}93 \\
(40.8)\end{array}$ & $\begin{array}{c}135 \\
(59.2)\end{array}$ & 7.737 & 0.005 \\
\hline $\begin{array}{l}\text { 7. SBL motivated me to do my best } \\
\text { work. }\end{array}$ & $\begin{array}{c}2 \\
(0.9) \\
\end{array}$ & $\begin{array}{c}30 \\
(13.2) \\
\end{array}$ & $\begin{array}{c}56 \\
(24.6) \\
\end{array}$ & $\begin{array}{c}88 \\
(38.6) \\
\end{array}$ & $\begin{array}{c}52 \\
(22.8) \\
\end{array}$ & $\begin{array}{c}88 \\
(38.6) \\
\end{array}$ & $\begin{array}{c}140 \\
(61.4) \\
\end{array}$ & 11.860 & 0.001 \\
\hline $\begin{array}{l}\text { 8. SBL has improved my analytic } \\
\text { skills. }\end{array}$ & $\begin{array}{c}5 \\
(2.2) \\
\end{array}$ & $\begin{array}{c}22 \\
(9.6)\end{array}$ & $\begin{array}{c}46 \\
(20.2) \\
\end{array}$ & $\begin{array}{c}101 \\
(44.3) \\
\end{array}$ & $\begin{array}{c}54 \\
(23.7) \\
\end{array}$ & $\begin{array}{c}73 \\
(32.0) \\
\end{array}$ & $\begin{array}{c}155 \\
(68.0) \\
\end{array}$ & 29.491 & $<\mathbf{0 . 0 0 0 5}$ \\
\hline $\begin{array}{l}\text { 9. SBL has promoted my ability to } \\
\text { work as a team member. }\end{array}$ & $\begin{array}{c}6 \\
(2.6) \\
\end{array}$ & $\begin{array}{c}36 \\
(15.8)\end{array}$ & $\begin{array}{c}48 \\
(21.1) \\
\end{array}$ & $\begin{array}{c}77 \\
(33.8)\end{array}$ & $\begin{array}{c}61 \\
(26.8)\end{array}$ & $\begin{array}{c}90 \\
(39.5)\end{array}$ & $\begin{array}{c}138 \\
(60.5) \\
\end{array}$ & 10.105 & 0.001 \\
\hline $\begin{array}{l}\text { 10. SBL produced a lot of pressure on } \\
\text { me as a student. }\end{array}$ & $\begin{array}{c}7 \\
(3.1) \\
\end{array}$ & $\begin{array}{c}23 \\
(10.1) \\
\end{array}$ & $\begin{array}{c}38 \\
(16.7) \\
\end{array}$ & $\begin{array}{c}107 \\
(46.9) \\
\end{array}$ & $\begin{array}{c}53 \\
(23.2) \\
\end{array}$ & $\begin{array}{c}68 \\
(29.8) \\
\end{array}$ & $\begin{array}{c}160 \\
(70.2) \\
\end{array}$ & 37.123 & $<\mathbf{0 . 0 0 0 5}$ \\
\hline $\begin{array}{l}\text { 11. SBL developed my planning } \\
\text { ability in work. }\end{array}$ & $\begin{array}{c}5 \\
(2.2) \\
\end{array}$ & $\begin{array}{c}25 \\
(11.0) \\
\end{array}$ & $\begin{array}{c}51 \\
(22.4) \\
\end{array}$ & $\begin{array}{c}92 \\
(40.4) \\
\end{array}$ & $\begin{array}{c}55 \\
(24.1) \\
\end{array}$ & $\begin{array}{c}81 \\
(35.5) \\
\end{array}$ & $\begin{array}{c}147 \\
(64.5) \\
\end{array}$ & 19.105 & $<\mathbf{0 . 0 0 0 5}$ \\
\hline $\begin{array}{l}\text { 12. SBL stimulated us to search for } \\
\text { various resources by ourselves. }\end{array}$ & $\begin{array}{c}9 \\
(3.9)\end{array}$ & $\begin{array}{c}28 \\
(12.3)\end{array}$ & $\begin{array}{c}41 \\
(18.0)\end{array}$ & $\begin{array}{c}98 \\
(42.9)\end{array}$ & $\begin{array}{c}52 \\
(22.8)\end{array}$ & $\begin{array}{c}78 \\
(34.2)\end{array}$ & $\begin{array}{c}150 \\
(65.8)\end{array}$ & 22.737 & $<\mathbf{0 . 0 0 0 5}$ \\
\hline $\begin{array}{l}\text { 13. Overall, I was satisfied with the } \\
\text { SBL method. }\end{array}$ & $\begin{array}{c}20 \\
(8.8)\end{array}$ & $\begin{array}{c}32 \\
(14.0)\end{array}$ & $\begin{array}{c}33 \\
(14.5)\end{array}$ & $\begin{array}{c}91 \\
(39.9)\end{array}$ & $\begin{array}{c}52 \\
(22.8)\end{array}$ & $\begin{array}{c}85 \\
(37.3)\end{array}$ & $\begin{array}{c}143 \\
(62.7)\end{array}$ & 14.754 & $<\mathbf{0 . 0 0 0 5}$ \\
\hline
\end{tabular}

SA: Strongly agree, A: Agree, U:Uncertain, DA: Disagree, SD: Strongly disagree, Data are presented as count and percentage, $\chi^{2}$ : Chi-square, $\mathrm{P}$ value is presented by one-sample chi-square test, $\mathrm{P}$ value $<0.05$.

Table 3. Students' feedback about teachers' role and guidance

\begin{tabular}{|l|c|c|c|c|c|c|c|c|c|}
\hline \multirow{2}{*}{ Domain } & \multicolumn{3}{|c|}{ Students' feedback (n= 228) } & \multicolumn{2}{|c|}{ Total } & \multicolumn{2}{c|}{ Significance } \\
\cline { 2 - 10 } & SD & $\mathbf{D}$ & $\mathbf{C}$ & $\mathbf{A}$ & SA & $\mathbf{D}$ & $\mathbf{A}$ & $\boldsymbol{\chi}^{\mathbf{2}}$ & $\boldsymbol{p}$ \\
\hline $\begin{array}{l}\text { 1. The teacher stimulated us to analyze } \\
\text { the scenario in a scientific way. }\end{array}$ & $\begin{array}{c}10 \\
(4.4)\end{array}$ & $\begin{array}{c}21 \\
(9.2)\end{array}$ & $\begin{array}{c}24 \\
(10.5)\end{array}$ & $\begin{array}{c}122 \\
(53.5)\end{array}$ & $\begin{array}{c}51 \\
(22.4)\end{array}$ & $\begin{array}{c}55 \\
(24.1)\end{array}$ & $\begin{array}{c}173 \\
(75.9)\end{array}$ & 61.070 & $<\mathbf{0 . 0 0 0 5}$ \\
\hline $\begin{array}{l}\text { 2. The teacher stimulated us to search } \\
\text { for links between scenario and the } \\
\text { discussed issue. }\end{array}$ & $\begin{array}{c}4 \\
(1.8)\end{array}$ & $\begin{array}{c}22 \\
(9.6)\end{array}$ & $\begin{array}{c}34 \\
(14.9)\end{array}$ & $\begin{array}{c}119 \\
(52.2)\end{array}$ & $\begin{array}{c}49 \\
(21.5)\end{array}$ & $\begin{array}{c}60 \\
(26.3)\end{array}$ & $\begin{array}{c}168 \\
(73.7)\end{array}$ & 51.158 & $<\mathbf{0 . 0 0 0 5}$ \\
\hline $\begin{array}{l}\text { 3. The teacher stimulated us to } \\
\text { summarize what we had learnt. }\end{array}$ & $\begin{array}{c}9 \\
(3.9)\end{array}$ & $\begin{array}{c}26 \\
(11.4)\end{array}$ & $\begin{array}{c}46 \\
(20.2)\end{array}$ & $\begin{array}{c}101 \\
(44.3)\end{array}$ & $\begin{array}{c}46 \\
(20.2)\end{array}$ & $\begin{array}{c}81 \\
(35.5)\end{array}$ & $\begin{array}{c}147 \\
(64.5)\end{array}$ & 19.105 & $<\mathbf{0 . 0 0 0 5}$ \\
\hline $\begin{array}{l}\text { 4. The teacher stimulated us to apply } \\
\text { knowledge to the discussed subject. }\end{array}$ & $\begin{array}{c}7 \\
(3.1)\end{array}$ & $\begin{array}{c}24 \\
(10.5)\end{array}$ & $\begin{array}{c}28 \\
(12.3)\end{array}$ & $\begin{array}{c}117 \\
(51.3)\end{array}$ & $\begin{array}{c}52 \\
(22.8)\end{array}$ & $\begin{array}{c}59 \\
(25.9)\end{array}$ & $\begin{array}{c}169 \\
(74.1)\end{array}$ & 53.070 & $<\mathbf{0 . 0 0 0 5}$ \\
\hline $\begin{array}{l}\text { 5. The teacher stimulated us to extract } \\
\text { cusses and inferences from the scenario. }\end{array}$ & $\begin{array}{c}6 \\
(2.6)\end{array}$ & $\begin{array}{c}12 \\
(5.3)\end{array}$ & $\begin{array}{c}37 \\
(16.2)\end{array}$ & $\begin{array}{c}112 \\
(49.1)\end{array}$ & $\begin{array}{c}61 \\
(26.8)\end{array}$ & $\begin{array}{c}55 \\
(24.1)\end{array}$ & $\begin{array}{c}173 \\
(75.9)\end{array}$ & 61.070 & $<\mathbf{0 . 0 0 0 5}$ \\
\hline
\end{tabular}

SA: Strongly agree, A: Agree, U:Uncertain, DA: Disagree, SD: Strongly disagree, $\chi^{2}$ : Chi-square, Data are presented as count and percentage, $P$ value $<0.05$.

$\mathrm{P}$ value is presented by one-sample chi-square test.

Data presented in Table 2 and Table 3 answered the second research question. The findings showed students' positive feedback regarding SBL strategy and teachers' role.

\section{Discussion}

Competent, safe nursing practice element is the main concern in nursing and health care specialties teaching [35]. Critical care nursing students may have limited opportunities to work independently [24]. They have short duration lectures and clinical practice. Thereby, the current study is an existing wealth vision about the use of
SBL strategy as part of nursing students' theoretical bulk of critical care nursing course.

\subsection{Students’'Achievement}

The current findings revealed that the majority of nursing students recorded high achievement scores regarding scenario inquiry, presentation performance, communication and language skills and harmony of team work. Additionally, their intellectual skills and usage of learning resources were achieved with moderate level. However, low achievement level of students was noted in critical appraisal and writing criteria domains. Overall, 
students reported achievement level ranged from good to excellent for all domains. This could be because students used their analytic skills effectively and delivered their outcome with high scores through constructive discussion and team work.

The current findings supported by Soliman, et al., [29], who adopting problem based leaning education approach in community nursing course for postgraduate nursing students. They noted that students' performance was very good in most achievement items. In addition, Amorim, et al., [14], recorded good medical students' performance in pediatric emergency department after simulation scenario application.

However, Abd El-Raouf, \& Ahmed [36], reported an upgrading in students' learning capabilities, critical thinking and problem solving talents and group collaboration. The same findings were depicted by Othman \& Anwar [30] and Abdou \& Dogham [24], in critical care nursing course. In the same view, some authors reported that scenario-based education expand students' knowledge and awareness and improve their leaning abilities and analytic skills [1,37].

Moreover, our findings concur with other investigators who revealed higher performance and information in intervention participants than control participants [18,28,32,38-40]. Other studies documented significant improvement in students' achievement regarding clinical and critical thinking, decision making skills and self-confidence after SBL application [16,24,41-44].

Parallel to our findings, Teixeira, et al., [45], noticed that simulation scenario improves students' engagement sessions analysis and discussion leading to effective clinical thinking and technical skills and competency for both students and teachers. While, Tocher \& Smith [12], added that SBL helped their students to use the learning resources properly and work as a team. Findings noted by Hursen, \& Fasli [2] Yeh [27] and Sorin [46], appointment that implementation of SBL can narrow the gap between theory and practice. In the contrary, some other studies noted that simulation scenario training limit students' achievement through acquiring minimum knowledge level of technical and non-technical compared with actual practice setting training $[15,47]$. According to other literature, application of simulation scenario did not affect students' score significantly especially critical thinking $[13,48,49]$ because its changes needs prolonged times [50,51].

Regarding communication skills two research studies showed that simulated SBL can improve nursing communication tools [18,52]. Also, two researchers stated that simulation scenario assisted nursing students to boost their communication skills in relation to reporting, recording, patient teaching and team work [21,53]. However, Hsu, et al [32], noted that nurses' communication performance improved in intervention group than the control group with significant difference between them after scenario based education. Conversely, other investigators reported lacking in communication skills among newly graduated nurses when facing real patients or their caregivers [13,54]. Lastly, the recent studies depicted that the SBL strategy produced a considerably positive effect on the students' achievement $[2,14,55]$.

\subsection{Students’ Feedback}

The present findings showed students' positive feedback regarding SBL and their teachers' guidance. Most of them reported that SBL was motive and enjoyable but very stressful. Also, about two third of them were satisfied with this teaching method because it boosted their technical and non-technical skills. These results are consistent with recent studies which reported that simulation scenario motivated most of nursing student in intervention group to learn with a valuable learning experience and competent clinical skills $[13,24,53,56]$. Similarly to our results some researchers found that students were unfamiliar and anxious with problem based scenario but enjoyed with this experience $[29,57,58]$. On the contrary, other investigators noted that problem based scenario was less stressful and more enjoyable for the majority of nursing students in different courses [30,31,53].

According to pervious and novice studies, overall students' satisfaction about SBL strategy and teachers' role and guidance were noted among the majority of studied students [16,32,41,53]. Also, Seybert, et al., [59], found high satisfaction and confidence among pharmacy students', after application of simulation scenario exercise for a pharmacotherapy course.

Moreover, other studies assured that enrolled student feedback to SBL was very positive with high learning experience enjoyment and they concluded that students perceived SBL as a valuable learning experience [14,60,61]. Likewise, Uysal [28], added that the students' positive feedback can be a map for developing and enhancing nursing curriculum each semester.

In general, application of SBL may lead to favorable safe patient care in critical care units [18]. Additionally, Abd El-Raouf, \& Ahmed [36], added that clinical simulated-based scenario improves the clinical teaching and learning processes and promotes an overall improvement and effectiveness of clinical learning. In the same view, other researchers concluded that simulation scenario is a creative teaching strategy that stimulates active learning with great potential technical proficiency and aggregates self-confidence [27,62,63].

\section{Conclusion}

Data of the current study supports the argument that SBL is beneficial and a dynamic learning strategy in critical care nursing education. It is easy to be conducted and it enhances students' appraisal skills and learning abilities. Additionally, it improves nursing students' critical thinking skills, self- confidence and their analytical skills. Thus, it helps them to bridge the gap between theory and practice. Hence, SBL could be a favorably integrated in nursing curriculum. Further studies are required to assess the effectiveness of this strategy on nursing students' achievement through the whole critical care nursing course. Another research study should be applied in parallel courses of the same level and different levels.

\subsection{Limitations of the study}

The current study is an initial one especially in critical care nursing course. One group of students was involved 
without a control group to investigate the efficacy of SBL and acquiring skills. It did not cover the practical parts related to the used topics. Also, the study was carried out only on fourth level students in one semester at one Faculty of Nursing in Egypt. Therefore, the generalization of the results will be very limited. Also, teachers' feedback regarding adopting SBL was not collected through this study.

\section{Acknowledgements}

The author wishes to acknowledge Faculty of Nursing, Mansoura University for facilitating this study. I would like also to acknowledge all staff members of critical care nursing department who involved in this study. Also, i would like to thank fourth level nursing students who participated in this study work and make it possible.

\section{Funding}

No financial support from any funding institution the author was received for his research or its publication.

\section{Conflict of Interest}

The author declares that he has no competing interest.

\section{References}

[1] Din, A. M., \& Jabeen, S. Scenario-based assessment exercises and the perceived learning of mass communication students. Asian Association of Open Universities Journal. 9(1): 93-103. (2014).

[2] Hursen, C., \& Fasli, F. G. Investigating the efficiency of scenario based learning and reflective learning approaches in teacher education. European Journal of Contemporary Education. 6(2): 264-279. 2017.

[3] Özçınar, H. Mapping teacher education domain: A document cocitation analysis from 1992 to 2012. Teaching and Teacher Education. 47: 42-61. 2015.

[4] Selmo, L., \& Orsenigo, J. Learning and sharing through reflective practice in teacher education in Italy. Procedia - Social and Behavioral Sciences, 116: 1925-1929. 2014.

[5] Skaalvik, E.M., \& Skaalvik, S. Teacher self-efficacy and teacher burnout: A study of relations. Teaching and Teacher Education. 26, 1059-1069. 2010.

[6] Huffman, J. L., McNeil, G., Bismilla, Z., \& Lai, A. Essentials of scenario building for simulation- based education. Comprehensive Healthcare Simulation: Pediatrics. 2016.

[7] Ku, T. K., \& Ha, M. The application of problem based learning in undergraduate nursing education: A strategy for curriculum reform. Journal of Biosciences and Medicines. 4: 52-59. 2016.

[8] Nagle, B. M., McHale, J. M., Alexander, G. A., \& French, B. M. Incorporating scenario-based simulation into a hospital nursing education program. J Contin Educ Nurs. 40(1):18-25. 2009.

[9] Alkaissi, A. Problem based learning in critical care nursing course: The students' experiences, a qualitative study. Conference: Fourth International Nursing Conference: "The Tipping Point: Creative Solutions to Health and Nursing Challenges". Two oral presentations: At: Amman - Jordan. January 2013.

[10] Akins, M., \& Crichton, S. Scenario Based Learning - Geography in the Field Using GIS / GPS for Curriculum Integration. National Educational Computing Conference. Washington, USA. 2003.

[11] Errington, E. P. Mission possible: Using near-world scenarios to prepare graduates fort the professions. International Journal of Teaching and Learning in Higher Education. 23(1): 84-91. 2011.
[12] Tocher, J. M., \& Smith, G. D. The experience of scenario-basedlearning in undergraduate nurse education in Edinburg. Macau Journal of Nursing. 7(2): 33-39. 2008.

[13] Jung, D., Lee, S. H., Kang, S. J., \& Kim, J. H. Development and evaluation of a clinical simulation for new graduate nurses: A multi-site pilot study. Nurse Education Today. 49: 84-89. 2017.

[14] Amorim, P. G., Morcillo, A. M., Fraga, A. M., Brandão, M. B. Belluomini, F., \& Baracat E. C. Realistic simulation in pediatric emergency: evaluation of strategy as a teaching tool for medical students. Arch Emerg Med Crit Care. 3(1): 1036. 2018.

[15] Persico, L. A Review: Using simulation-based education to substitute traditional clinical rotations. JOJ Nurse Health Care. 9(3): 555762. 2018.

[16] D’Souza, M. S., Venkatesaperumal, R., Chavez, F. S., Parahoo, K., \& Jacob, D. Effectiveness of simulation among undergraduate students in the critical care nursing. International Archives of Nursing and Health Care. 3(4): 2017.

[17] Edgecombe, K., Seaton, P., Monahan, K., Meyer, S., LePage, S., \& Erlam, G. Clinical simulation in nursing: A literature review and guidelines for practice. Ako Aotearoa, National Centre for Tertiary Teaching Excellence. June 2013. http://akoaotearoa.ac.nz/communities/collaboration-clinicalsimulation-leading-way.

[18] Rahmani, A., Mohammadi, A., \& Moradi, Y. Effectiveness of scenario-based education on the performance of the nurses in the critical cardiac care unit for patients with acute coronary syndrome. International Journal of Medical Research \& Health Sciences. 5(8): 218-224. 2016.

[19] Valler-Jones, T., Meechan, R., \& Jones, H. Simulated practice: A panacea for health education? British Journal of Nursing. 20(1): 628-631. 2011.

[20] Murray, C., Grant. M., Howarth, M., \& Leigh, J. The use of simulation as a teaching and learning approach to support practice learning. Nurse Education in Practice. 8: 5-8. 2008.

[21] Battista, A. An activity theory perspective of how scenario-based simulations support learning: a descriptive analysis. BioMed Central. 2:23 2017.

[22] ACARA. Australian Curriculum Assessment and Reporting Authority. General capabilities in the Australian Curriculum. 2012.

http://www.australiancurriculum.edu.au/GeneralCapabilities/Gene ral\%20capabilities.

[23] Barrie, S. Rethinking generic graduate attributes. HERDSA News 27, 1-6. 2005. http://www.herdsa.org.au/wpcontent/uploads/2007/06/1_herdsa_news_april_2005.

[24] Abdou, F. A., \& Dogham, R. S. Impact of Using Simulation on Critical Care Nursing Students' Knowledge and Skills of Acute Respiratory Distress Syndrome. IOSR Journal of Nursing and Health Science. (5): 28-425. 2016.

[25] Bultas, M., Hassler, L., Ercor, N., \& HaasF, .Enhancing the pediatric undergraduate nursing curriculum through simulation. Journal of Pediatric Nurse. 2013; 26(3):224-9.

[26] Thomsen, B. C., Renaud,C. C., Savory, S. J., Romans, E. J., Mitrofanov, O., Rio, M. et al. Introducing scenario based learning: Experiences from an undergraduate electronic and electrical engineering course. Conference Paper · May 2010.

[27] Yeh, Y. C. Undergraduate nursing students' perceptions of high fidelity simulation-based learning. International Archives of nursing and Health Care. 2(3): 2016.

[28] Uysal, N. Improvement of nursing students' learning outcomes through scenario-based skills training. Rev. Latino-Am. Enfermagem. 24:e2790; 2016.

[29] Soliman, S. M., Abd El -Mouty, S. M., \& Salem, N. M. Postgraduate nursing students' acquire skills upon applying problem based learning. IOSR Journal of Nursing and Health Science. 6(2): 05-11. 2017.

[30] Othman, S. Y., \& Anwar S. S. Students' perception and acceptance of problem-based learning approach in critical care nursing practice. Scientific Cooperation International Workshops on Medical Topics, Ankara-TURKEY. 52-67. 2014.

[31] Rhodes, M. How Two Intuitive Theories Shape the Development of Social Categorization? Child Development Perspective. 7(1): 12-16. 2013.

[32] Hsu, L. L., Chang, W. H., \& Hsieh, S.I. The effects of scenariobased simulation course training on nurses' communication 
competence and self-efficacy: a randomized controlled trial. Journal of Professional Nursing. 31(1): 37-49. 2015.

[33] Clark, C. M., Faan, A., Ahten, S. M., \& Macy, R. Using problem-based learning scenarios to prepare nursing students to address incivility. Clinical Simulation in Nursing. 9, e75-e83. 2013.

[34] Tosterud, R. Simulation used as a learning approach in nursing education: Students' experiences and validation of evaluation questionnaires. Faculty of Health, Science and Technology, Gjøvik University College. Karlstad University Studies. 2015:1.

[35] $\mathrm{Li}, \mathrm{A}$. M. Simulation-based clinical skill training to promote effective clinical learning with simulation evaluation rubrics in nursing education. International Journal of Information and Education Technology. 6(3): 237-242. 2016.

[36] Abd El-Raouf, S. E., \& Ahmed, A. I. Nursing students' experiences with problem based learning: a teaching strategy applied in community health course med. J. Cairo Univ.79(1): 323-333. 2014.

[37] Mikkelsen, J., Reime, M. H., \& Harris, A. K. Nursing students' learning of managing cross-infections - scenario-based simulation training versus study groups. Nurse Education Today. 28, 664-671. 2008.

[38] Staun, M., Bergström, B., \& Wadensten, B. Evaluation of a PBL strategy in clinical supervision of nursing students: Patient-centred training in student-dedicated treatment rooms. Nurse Education Today. 30(7):631-7. 2010.

[39] Devlin, J. W., Marquis, F., Riker, R. R., Robbins, T., Garpestad, E., Fong, J. J., et al. Combined didactic and scenario-based education improves the ability of intensive care unit staff to recognize delirium at the bedside. Critical Care. 12(R19): 2008.

[40] Nasef, M. M., El-Tayeb, I. M., Mawgoud, R. A., \& Hammam, D. A. Active learning versus lecture-based approaches in occupational training programs of intensive care nursing staff at Al-Ahrar Hospital, Zagazig City, Egypt. Gaziantep Medical Journal. 20(2):147-59. 2014.

[41] Jeffries, P., \& Rizzola, M. Designing and implementing models for the innovative use of simulation in teaching nursing care of ill adults and children: A national, multi-site, multi-method study. New York, NY: National League for Nursing. 2012. Retrieved from:www.nln.org/research/LaerdalReport.

[42] Bambini, D., Washburn, J., \& Perkins, R. Outcomes of clinical simulation for novice nursing students: Communication, confidence, clinical judgment. Nursing Education Perspectives. 30(2):79-82. 2013.

[43] Nevin, M., Neill, S., \& Mulkerrins, N. Measuring student perceptions of clinical competence. Journal of Nurse Education. 42(12):548-54. 2014

[44] Kaddoura, M. New graduate nurses' perceptions of the effects of clinical simulation on their critical thinking, learning, and confidence. Journal of Continuing Education in Nursing. 41(11):506-16. 2014

[45] Teixeira, C. R., Pereira, M. C., Kusumota, L., Gaioso, V. P., Mello, C. L., \& de-Carvalho, E. C. Evaluation of nursing students about learning with clinical simulation. Rev Bras Enferm. 68(2): 284-91. 2015.

[46] Sorin, R. Scenario-based learning: Transforming tertiary teaching and learning. 2013 Retrieved: form http://researchonline.jcu.edu.au/30512/3/30512\%20Sorin\%202013.
[47] Alinier, G., Hunt, B., Gordon, R., \& Harwood, C. Effectiveness of intermediate-fidelity simulation training technology in undergraduate nursing education. Journal of Advanced Nursing. 54(3):359-369. 2006.

[48] Maneval, R., Fowler, K. A., Kays, J. A., Boyd, T. M., Shuey, J., Harne-Britner, S., et al. The effect of high-fidelity patient simulation on the critical thinking and clinical decision-making skills of new graduate nurses. J. Contin. Educ. Nurs. 43:125-134. 2012.

[49] Shinnick, M. A.,\& Woo, M. A. The effect of human patient simulation on critical thinking and its predictors in prelicensure nursing students. Nurse Education Today. 33: 1062-1067. 2013.

[50] Costley, J., \& Han, S. L. Critical thinking and interaction changed over time: a study of an asyhchronous forum. Adv. Sci. Technol. Lett. 36:78-82. 2013.

[51] Wong, M. S. A prospective study on the development of critical thinking skills for student prosthetists and orthotists in Hong Kong. Prosthet. Orthot. Int. 31(2):138-146. 2007.

[52] Nestel, D., Kneebone, R., \& Kidd, J. Teaching and learning about skills in minor surgery. Journal of Clinical Nursing. 12 (2): 291-6. 2003.

[53] ALFozan, H., El Sayed, Y., \& Habib, F. Designing, Implementing and Evaluating Preclinical Simulation Lab for Maternity Nursing Course. Journal of Education and Practice. 6(12): 152-162. 2015.

[54] Sönmez, B., \& Yildirim, A. Difficulties experienced by newlygraduated nurses in Turkey: a qualitative study of the first six months of employment. J. Nurs. Educ. Pract. 6,104. 2016.

[55] Özsevgeç, L. C., \& Kocadağ, Y. The effects of scenario based learning approach to overcome the students'misconceptions about inheritance. H. U. Journal of Education. 28(3): 83-96. 2013.

[56] Oh, P. J., Jeon, K. D., \& Koh, M. S. The effects of simulationbased learning using standardized patients in nursing students: a meta-analysis. Nurse Education Today. 35:e6-e15. 2015.

[57] Yuans, H., Williams, A. B., Yinl, Liu, M., Fang, B. J. \& Pang, D. Nursing students' views on the effectiveness of problem-based learning China. IN Press Nurse Education Today. 2010.

[58] Flint, S., \& Stewart, T. Food microbiology - design and testing of a virtual laboratory exercise. Journal of Food Science Education. 9(4): 84-89. (October, 2010).

[59] Seybert, A. L., Laughlin, K. K., Benedict, N. J., Barton, C. M., Rea, R. S. Pharmacy student response to patientsimulation mannequins to teachperformance-based pharmacotherapeutics. Am J Pharm Educ. 70(3): 2006.

[60] Blackburn, G. Innovative eLearning: Technology shaping contemporary problem based learning: a cross-case analysis. Journal of University Teaching \& Learning Practice. 12(2): 1-17. 2015.

[61] Feingold, C. E., Calaluce, M., \& Kallen, M. A. Computerized patient model and simulated clinical experiences: Evaluation with baccalaureate nursing students. The Journal of Nursing Education. 43: 156-163. 2004.

[62] Chen, S. L., Lee, M. L., Liao, I. C., Liang, T. The development and current status of high fidelity patient simulation teaching. $\mathrm{Hu}$ Li Za Zhi. 60: 87-92. 2013.

[63] Taplay, K., Jack, S. M., Baxter, P., Eva, K., Martin, L. The process of adopting and incorporating simulation into undergraduate nursing curricula: a grounded theory study. $J$ Prof Nurs. 31: 26-36. 2015. 